\title{
Zooplâncton do estuário do Pina (Recife-Pernambuco-Brasil): composição e distribuição temporal
}

\author{
Encida Maria ESKINAZI-SANT'ANNA ${ }^{1}$ \& José Galíria TUNDISI ${ }^{2}$ \\ ${ }^{1}$ Instituto de Biociências da Universidade de São Paulo \\ Departamento de Ecologia Geral \\ (Caixa Postal 11461, 05422-970 São Paulo, SP, Brasil) \\ ${ }^{2}$ Escola de Engenharia de Sāo Carlos da Universidade de Sāo Paulo \\ Centro de Recursos Hídricos e Ecologia Aplicada \\ (Av. Dr. Carlos Botelho, 1475, 13560-250, Sāo Carlos, SP, Brasil)
}

\begin{abstract}
- Abstract: Composicion and daily variation of zooplankton in the estuary of Pina (Brazil-PE) was studied based on 200L of water filtered at surface through a net mesh size of $69 \mu \mathrm{m}$, during the dry (January-February/91) and the rainy (June-July/91) seasons. The samples were collected with time interval of three hours, during a daylight period. Concurrent hydrological data were obtained. Nineteen taxon of zooplankton were registered, including meroplankton, of which the most abundant was Favella ehrenbergi, followed by Brachionus plicatilis, Copepoda nauplii and Oithona osvaldocruzi (Q). Higher quantitatives values occured in the dry şeason. The maximum registered was $411,749 \mathrm{org} \cdot \mathrm{m}^{3}$ and the minimum $2,623 \mathrm{org} \cdot \mathrm{m}^{-}$. The tidal currents were the main factor influencing the zooplankton spacial variation. Specific diversity was low, highlighting selective processes in the Pina estuary.
\end{abstract}

- Resumo: Estudos sobre a composiçāo e variação diurna do zooplâncton do estuário do Pina (PE) foram realizados, baseando-se em 200 litros de água superficial, coletada e filtrada em uma rede com malha de $69 \mu \mathrm{m}$ de abertura, durante os períodos seco (janeiro e fevereiro/91) e chuvoso (junho e julho/91), em um ciclo diurno de $12 \mathrm{~h}$. Paralelamente, foram coletados dados hidrológicos. (O zooplâncton esteve representado por 19 taxa, alćm do meroplâncton. Destacaram-se Favella eltrenbergi, Brachionus plicatilis, náuplios de Copepoda e Oithona osvaldocruzi (Q). Valores quantitativos mais altos foram registrados no período seco. O máximo registrado foi de $411.749 \mathrm{org} \cdot \mathrm{m}^{-3}$ e o mínimo $2.623 \mathrm{org} \cdot \mathrm{m}^{-3}$. As correntes de marés foram as principais responsáveis pela variação espacial do zooplâncton. A diversidade específica foi baixa, evidenciando processos seletivos no estuário.

- Descriptors: Zooplankton, Estuary, Community composition, Diurnal and seasonal variation, Species diversity, Brazil.

- Descritores: Zooplâncton, Estuário, Composição, Variação diurna e sazonal, Diversidade de espécies, Brasil.

\section{Introdução}

Os estuários são importantes ecossistemas por serem do ponto de vista biológico, altamente produtivos. São ambientes muito dinâmicos, onde os fatores físicos, químicos e biológicos sofrem constantes modificações. Essa complexidade ecossistêmica resulta em um "pool" genético extremamente diverso, com a biota originada dos ambientes terrestre, marinho e de água doce, além de uma fauna e flora autóctones.
O estuário do Pina situa-se na zona urbana do Recife, próximo ao porto da cidade. A área recebe despejos de efluentes domésticos e industriais, o que compromete a qualidade de suas águas e de sua biota, a qual é utilizada como fonte de subsistência para inúmeras familias.

$O$ estudo das variaçōes diurnas e sazonais do zooplâncton em um ambiente tão impactado, torna-se necessário, pois o conhecimento da composição e abundância da comunidade zooplanctônica pode ser utilizado para a caracterização e previsão de alterações 
ambientais induzidas ou não (Parsons et al., 1984; Soetaert \& Van Rijswijk, 1993).

Trabalhos sobre as variaçōes temporais do zooplâncton são importantes, uma vez que as mudanças que ocorrem entre populações em um estuário, mesmo em curto espaço de tempo, têm magnitude para afetar não apenas as populações, mas todo o ecossistema (Checkley et al., 1992). Além disso, estudos dessa natureza contribuem para um melhor entendimento das interrelaçōes do zooplâncton com o meio, além de servirem como base para estimativa da produtividade dos ambientes estuarinos.

Em estuários do nordeste são poucos os trabalhos sobre variação diurna do zooplâncton. Neumann-Leitão et al. (1992 a e b) iniciaram recentemente esse tipo de abordagem, em estuários do rio Massangana e Tatuoca, em Suape (PE).

No estuário do Pina, alguns trabalhos foram desenvolvidos por Feitosa (1988), Silva-Cunha et al. (1990) ${ }^{1}$ e Silva \& Mello $(1991)^{2}$, com relação à composição e biomassa fitoplanctônica, parâmetros hidrológicos e malacofauna.

O presente trabalho foi desenvolvido para fornecer informações sobre a composição e distribuição temporal do zooplâncton, em relação aos ciclos diurnos de marés e durante diferentes períodos sazonais.

\section{Material e métodos}

O estuário do Pina localiza-se na parte interna do porto da cidade do Recife (PE), sendo formado pela confluência dos rios Capibaribe (através do seu braço sul), Tegipió, Jordão e Pina. Sua área total é de $3,6 \mathrm{~km}^{2}$, com larguras entre 0,26 e 0,86 km (CONDEPE, 1980) (Fig. 1).

A área é um ambiente dinâmico do ponto de vista hidrográfico, com salinidades variando entre 0,42 a $37,00 \mathrm{e}$ a temperatura da água entre 24,00 e $32,00^{\circ} \mathrm{C}$, com profundidade máxima de $4,50 \mathrm{~m}$. O regime de marés é semi-diurno, com amplitude máxima de $3,5 \mathrm{~m}$ durante a maré alta (Coimbra et al., $1987^{3}$; Feitosa, 1988).

(1) Silva-Cunha, M. G. G.; Feitosa, F. A.; Almeida, C. D. P. 1990. Diatomáceas da Bacia do Pina (PE). In: REUNIÄO NORDESTINA DE BOTÂNICA, XIV. Resumos. Recife, UIPE, p.28.

(2) Silva, J. A. B. \& Mello, R. L. S. 1991. Informaçōes preliminares sobre a malacofauna do rio Capibaribe e Bacia do Pina, Recife-PE. In: CONGRESSO NORDESTINO DE ECOLOGIA, IV. Resumo. Recife, Sociedade Nordestina de Ecologia. p. 17.

(3) Coimbra, A. L. S.; Costa, K. M. P.; Macedo, S. J. 1987. Estudo ecológico da Bacia do Pina. Características físico-químicas da água. In: ENCONTRO BRASILEIRO DE OCEANOGRAFIA QUIMICA. Resumo. Recife, UFPE p. 21.
As colctas foram realizadas cm uma estaçāo fixa, nos meses de janeiro (dia 22) e fevereiro (dia 15), no período seco, e junho (dia 28) e julho (dia 03), durante o período chuvoso, em 1991. A estação de coleta situa-se na regiāo central do estuário do Pina, no principal canal de navegação da área. As amostragens foram diurnas (das 05:00 às 17:00 h) e realizadas de $3 \mathrm{em} 3$ horas, abrangendo o total de 12 horas.

A transparência da água foi obtida através do lançamento do disco de Secchi, até o limite máximo de visualização. Para o cálculo do coeficiente de extinção da luz, utilizou-se a fórmula de Poole e Atkins (1929).

As amostras para análise das variáveis físicas e químicas foram coletadas em três profundidades da coluna d'agua: superfície, meio e fundo, utilizando-se garrafas de Nansen. Após a coleta, as subamostras foram acondicionadas em frascos de $150 \mathrm{ml}$ para posterior análise.

A temperatura da água foi obtida através de leitura direta no termômetro de reversão, acoplado à garrafa de Nansen. A salinidade foi determinada através do método de Mohr-Knudsen, descrito em Strickland \& Parsons (1965).

O oxigênio dissolvido foi obtido por titulação, segundo o método de Winkler em Strickland \& Parsons (1965). A porcentagem de saturação do oxigênio foi calculada segundo as tabelas do National Institute of Oceanography of Great Britain and Unesco (UNESCO, 1973). Para a determinação da Demanda Bioquímica de Oxigênio (DBO), foi utilizada a técnica descrita no Standard Methods for Examination of Water and Wastewater (APHA, 1985). O pH foi medido através de potenciômetro Beckman Zeromatic II.

As amostras superficiais de plâncton foram obtidas, filtrando-se 200 litros de água através de um tubo de PVC acoplado a uma tela de nylon de $69 \mu \mathrm{m}$ de abertura de malha. Apos a coleta, as amostras foram fixadas com formol neutro a $4 \%$.

Para cada amostra, subamostras de $4 \mathrm{ml}$ foram analisadas. Em caso de pouca representatividade, toda a amostra era inspecionada.

A diversidade de espécies foi calculada utilizando-se o índice de Shannon (1948), e o agrupamento das espécies pelo coeficiente de Sorensen (1948), através do método de agrupamento por WPGMA. Análises estatísticas de correlação entre variáveis bióticas e abióticas foram também realizadas. 


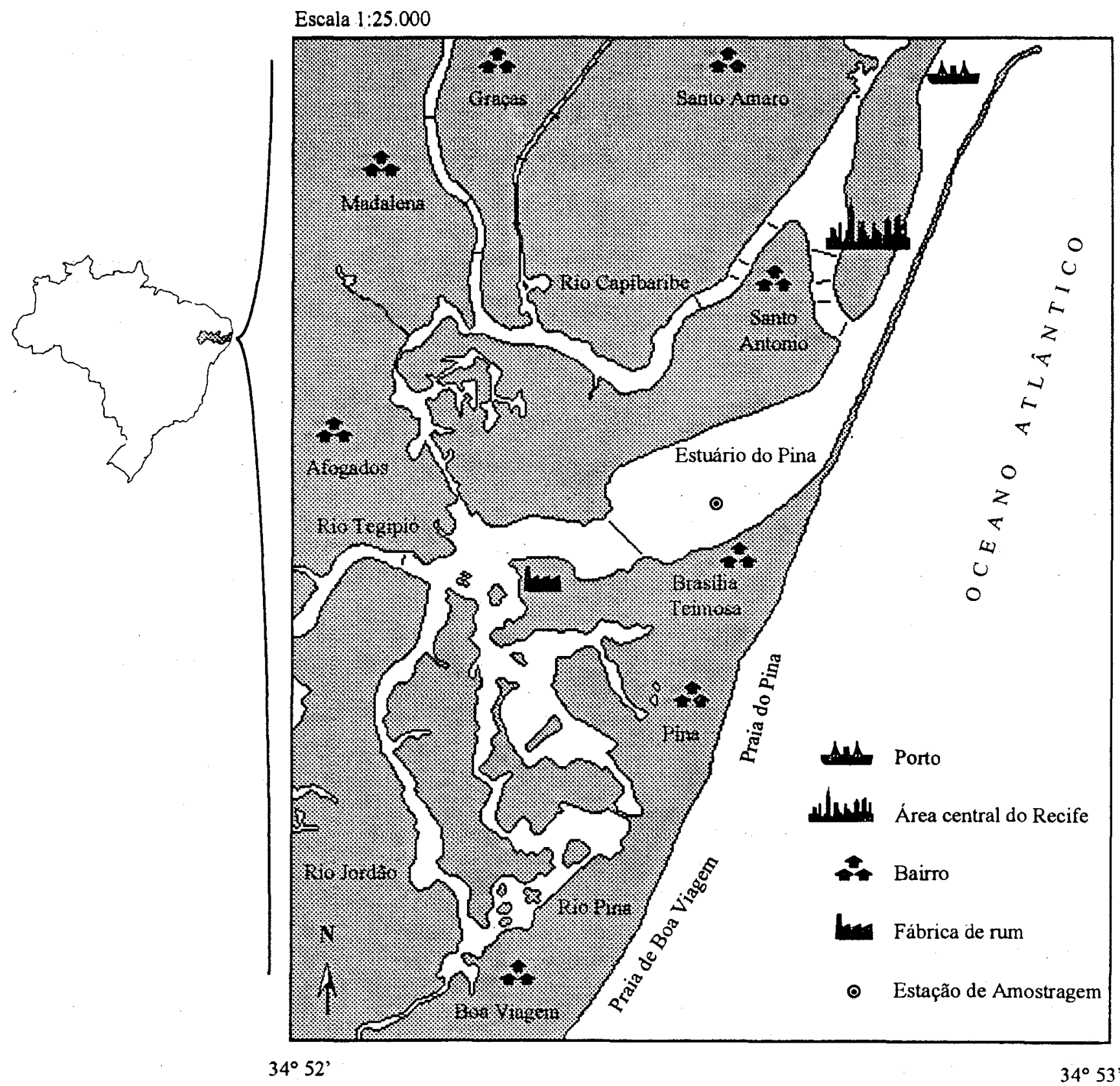

Fig. 1. Mapa da área estudada e localização da estação de amostragem.

\section{Resultados}

\section{Transparência da água}

A transparência da água apresentou valores reduzidos (máximo de $1,90 \mathrm{~m}$ em junho, correspondendo a um coeficiente de extinção de luz de $0,89 \mathrm{~m}$ ). Houve redução significativa da transparência da água no mês de julho (período chuvoso), onde foram registrados os menores valores $(0,30$ e $0,40 \mathrm{~m}$, correspondendo a 5,66 e $4,25 \mathrm{~m}$ de coeficiente de extinção de luz, respectivamente). Durante o período seco, os valores de transparência da água mantiveram-se em torno de $1,00 \mathrm{~m}$ e o coeficiente de extinção de luz acima de $1,00 \mathrm{~m}$, com exceção das amostragens realizadas às 5 e 8:00 h (marés enchente $\mathrm{e}$ cheia, respectivamente), quando foram registrados valores da ordem de $0,01 \mathrm{~m}$.

\section{Temperatura da água}

A temperatura da água apresentou pequenas variações, registrando-se as temperaturas mais baixas na 
profundidade máxima local $\left(24,90^{\circ} \mathrm{C}\right)$, e mais clevadas na camada superficial da coluna d'agua $\left(30,90^{\circ} \mathrm{C}\right)$.

Ocorreu uma pequena variação sazonal da temperatura na coluna d'água, encontrando-se as temperaturas mais elevadas durante o período seco (máxima de $30,90^{\circ} \mathrm{C}$ e mínima de $27,50^{\circ} \mathrm{C}$ ), enquanto que no período chuvoso, as temperaturas foram um pouco mais reduzidas (máxima de 27,90 e mínima de $24,80^{\circ} \mathrm{C}$ ).

\section{Salinidade}

A salinidade na coluna d'agua apresentou uma estratificação acentuada, sendo encontrados os maiores valores nas preamares $(>30,00)$ e nas camadas mais profundas. As amplitudes de salinidade nos períodos seco e chuvoso foram bastante acentuadas, podendo-se observar durante o período chuvoso, a influência marcante dos aportes continentais, acarretando valores de salinidade mais reduzidos (máxima de 22,48 e mínima de 5,39). Durante o período seco, a salinidade máxima registrada foi de 33,70 e a mínima de 12,5 .

\section{Oxigênio dissolvido}

De uma maneira geral, os teores de oxigênio dissolvido foram reduzidos em ambos os períodos sazonais, com valores médios em torno de $4,0 \mathrm{ml} . \mathrm{I}^{-1}$, com saturação máxima de 191,15\%. O valor máximo registrado foi de 9,29 ml..$^{-1}$, no período chuvoso (maré enchente) e o mínimo de $1,67 \mathrm{mll}^{-1}$ no período seco (maré cheia).

As variações no teor de oxigênio dissolvido estiveram relacionadas principalmente com as correntes de marés, especialmente durante o período chuvoso, quando o volume de água de origem continental no interior do estuário aumenta, propiciando a formação de áreas de grande turbulência, influenciando assim os níveis de oxigênio dissolvido na coluna d'água. O oxigênio dissolvido não apresentou estratificação vertical significativa, observando-se valores relativamente homogêneos para a coluna d'agua durante as diferentes marés.

\section{D.B.O.}

No período seco, os teores mais elevados de D.B.O. foram registrados durante a baixa-mar (valor máximo de $5,67 \mathrm{mg} . \mathrm{l}^{-1}$ e mínimo de $0,88 \mathrm{mg} . \mathrm{l}^{-1}$ ), enquanto no período chuvoso, a D.B.O. apresentou seus valores máximos durante as preamares (máxima de $5,21 \mathrm{mgl}^{-1} \mathrm{e}$ mínima de

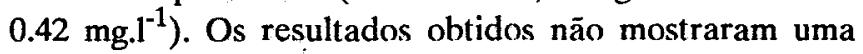
variação sazonal significativa da D.B.O.

\section{pH}

$\mathrm{O}$ pH variou de neutro a alcalino, apresentando no período seco, o máximo de 8,30 e o mínimo de 7,40. No período.chuvoso o valor máximo foi de 8,36 e mínimo de 7,21 .

\section{Zooplâncton (Tabs 1 e 2, Figs 2, 3 e 4)}

No estuário do Pina, Copepoda foi o grupo mais representativo da comunidade zooplanctônica, ocorrendo em todas as amostras estudadas. Neste grupo destacou-se Oithona osvaldocruzi (Q), como o único organismo que ocorreu em todas as amostras. Entre os organismos meroplanctônicos, destacaram-se como muito freqüentes na área, náuplios e cípris de Cirripedia e zoeas de Brachyura.

Alguns taxa ocorreram apenas no período chuvoso: larvas de Polychaeta, Moina sp, Eucalanus pileatus ( $\sigma^{*} \mathrm{e} Q$ ), Centropages velificatus ( $\left(\sigma^{x} \mathrm{e} Q\right.$ ), Notodiaptomus cearensis $\left(\sigma^{\top}\right)$, Pseudodiaptomus acutus ( $\sigma^{\star}$ e $\left.Q\right)$, Labidocera fluviatilis ( $\sigma^{\pi}$ e $\left.Q\right)$, Acartia lilljeborgi $\left(O^{*}\right)$ Hemicyclops thalassius ( $O^{x} \mathrm{e}$ Q) e Sagitta sp.

No período seco, o zooplâncton apresentou densidades elevadas (máximo de 411749 org.m ${ }^{-3}$ ), especialmente devido à significativa contribuição do holoplâncton, que constituiu mais de $70 \%$ do zooplâncton total, chegando a $9906 \%$ em janeiro (maré enchente).

Em termos de abundância relativa, os organismos mais representativos foram Favella ehrenbergi, Brachionus plicatilis, náuplio de Copepoda, Oithona hebes () e Oithona osvaldocruzi ( () . Favella ehrenbergi foi o organismo com maior contribuição percentual no período seco, chegando a 79,42\% do zooplâncton total em janeiro, durante a maré enchente $(05: 00 \mathrm{~h}) . O$. osvaldocruzi $(Q)$ também apresentou valores percentuais bastante significativos, chegando a constituir $41,93 \%$ do zooplâncton total em fevereiro (maré cheia).

O meroplâncton apresentou contribuiçōes quatitativas bastante reduzidas, com valores mais significativos nas amostras das 08:00 h em janeiro (preamar), e 05:00 h em fevereiro (preamar), em função da ocorrência expressiva de cípris de Cirripedia e zoeas de Brachyura. 
Tabela 1. Variação da densidade (org. $\mathrm{m}^{3}$ ), densidade (blts/ind) e eqüitabilidade zooplanctônica no estuário do Pina (PE), no perlodo seco (janeiro e fevereiro/91)

\begin{tabular}{|c|c|c|c|c|c|c|c|c|c|c|}
\hline \multirow[b]{2}{*}{ ORGANISMOS } & \multicolumn{5}{|c|}{ janeiro } & \multicolumn{5}{|c|}{ fevereiro } \\
\hline & $\begin{array}{c}\text { 05:00 } \\
\text { Enchente }\end{array}$ & $\begin{array}{c}\text { 08:00 } \\
\text { Preamar }\end{array}$ & $\begin{array}{c}11: 00 \\
\text { Vazante }\end{array}$ & $\begin{array}{c}\text { 14:00 } \\
\text { Baixa-mar }\end{array}$ & $\begin{array}{c}17: 00 \\
\text { Enchente }\end{array}$ & $\begin{array}{c}\text { 05:00 } \\
\text { Preamar }\end{array}$ & $\begin{array}{c}\text { 08:00 } \\
\text { Vazante }\end{array}$ & $\begin{array}{c}\text { 11:00 } \\
\text { Baixa-mar }\end{array}$ & $\begin{array}{c}\text { 14:00 } \\
\text { Enchente }\end{array}$ & $\begin{array}{l}\text { 17:00 } \\
\text { Preamar }\end{array}$ \\
\hline Centropyxis acureata & - & - & - & - & - & - & - & - & 187 & - \\
\hline Favella ehrenbergi & 49875 & 3375 & 41250 & 20250 & 66000 & 53625 & 39375 & 37500 & 26250 & 11812 \\
\hline Brachionus plicatilis & - & - & 937 & - & 1125 & ـ & - & 103125 & 15000 & 375 \\
\hline Larvas de Bivalvia & - & - & 562 & - & - & - & - & - & - & 1125 \\
\hline Larvas de Gastropoda & - & - & 750 & - & 187 & - & - & - & 750 & . \\
\hline Náuplios de Cirripedia & 562 & 1500 & 562. & 375 & 750 & 1500 & 375 & 2062 & 1125 & 2250 \\
\hline Cipris de Cirripedia & 1125 & 2250 & 375 & 937 & 187 & 750 & 937 & 1875 & 4875 & 187 \\
\hline Náuplios de Copepoda & 1500 & 1875 & 1687 & 562 & 26250 & 138750 & - & 6750 & 94500 & 10125 \\
\hline Paracalanus quasimodo $\left(\varnothing^{\infty}\right)$ & 375 & - & - & - & 187 & 937 & 187 & - & - & 375 \\
\hline Paracalanus quasimodo ( $($ ) & 1125 & 375 & 375 & - & 937 & 4125 & 1500 & - & - & 375 \\
\hline Paracalanus indicus $\left(0^{\circ}\right)$ & - & - & - & - & - & 750 & - & - & - & 375 \\
\hline Paracalanus indicus ( 8 ) & 562 & - & - & - & 562 & 2250 & 375 & - & - & 375 \\
\hline Paracalanus crassirostris ( $0^{\circ}$ ) & - & - & - & - & 187 & 3000 & 375 & - & - & - \\
\hline Paracalanus crassirostris ( $\bar{Q}$ ) & 1500 & 750 & 375 & 375 & 562 & 7125 & 1875 & . & 375 & 375 \\
\hline Acantia lilljeborgi (\$) & - & - & - & - & - & - & - & - & 375 & - \\
\hline Oithona osvaldocruzi $\left(\sigma^{\prime}\right)$ & 937 & 562 & 2250 & 375 & 3937 & 8437 & 4312 & 1125 & 3750 & 2812 \\
\hline Oithona osvaldocruzi ( $q)$ & 2625 & 3000 & 10312 & 1125 & 13125 & 67875 & 19500 & 2625 & 90750 & 26250 \\
\hline Oithona hebes ( $\left.\sigma^{\prime \prime}\right)$ & - & - & - & - & 1500 & - & 1500 & - & 375 & \\
\hline Oithona hebes ( $\$$ ) & 1125 & 1875 & $1875^{\circ}$ & 562 & 3750 & 26250 & 9375 & 2437 & 4687 & 2812 \\
\hline Euterpina acutifrons ( $\left.0^{\circ}\right)$ & - & - & - & 187 & - & - & - & 562 & - & 187 \\
\hline Euterpina acutifrons (Q) & 750 & 1125 & 750 & 937 & - & 2250 & 1500 & 1875 & 2250 & 1125 \\
\hline Larvas de Crustacea Decapoda & - & - & - & - & - & - & - & - & 375 & - \\
\hline Zoeas de Brachyura & 750 & 375 & 562 & 750 & - & 93750 & 375 & 750 & 1125 & 1500 \\
\hline Ovos de Peixes & - & - & - & - & - & - & , & - & 750 & 187 \\
\hline Larvas de Peixes & - & - & $\therefore$ & - & $=$ & 375 & - & - & - & - \\
\hline TOTAL HOLOPLANCTON & 60374 & 12937 & 59811 & 24373 & 118122 & 315374 & 79874 & 155999 & 238499 & 57373 \\
\hline TOTAL MEROPLÁNCTON & 2437 & 4125 & 2811 & 2062 & 1124 & 96375 & 1687 & 4687 & 9000 & 5249 \\
\hline TOTAL & 62811 & 17062 & 62622 & 26435 & 119246 & 411749 & 81561 & 160686 & 247499 & 62622 \\
\hline DIVERSIDADE (bits/ind) & 1.36 & 3.02 & 1,71 & 1.45 & 1.83 & 2.45 & 1.95 & 1.54 & 2.10 & 2.40 \\
\hline EQUITABILDADE & 0.39 & 0.91 & 0.46 & 0.45 & 0.53 & 0.68 & 0.59 & 0.50 & 0.54 & 0.63 \\
\hline
\end{tabular}

Tabela 2. Variação da densidade (org. $\mathrm{m}^{3}$ ), diversidade (bits/ind) e eqüitabilidade zooplanctônica no estuário do Pina (PE), no periodo chuvoso (junho e julho/91).

\begin{tabular}{|c|c|c|c|c|c|c|c|c|c|c|}
\hline ORGANISMOS & $\begin{array}{c}05: 00 \\
\text { Preamar }\end{array}$ & $\begin{array}{c}08: 00 \\
\text { Vazante }\end{array}$ & $\begin{array}{c}\text { junho } \\
\text { 11:00 } \\
\text { Baixa-mar }\end{array}$ & $\begin{array}{c}14: 00 \\
\text { Enchente }\end{array}$ & $\begin{array}{c}\text { 17:00 } \\
\text { Preamar }\end{array}$ & $\begin{array}{c}08: 00 \\
\text { Enchente }\end{array}$ & $\begin{array}{l}08.00 \\
\text { Pregmar }\end{array}$ & $\begin{array}{l}\text { jutho } \\
\text { 11:00 } \\
\text { Vazante }\end{array}$ & $\begin{array}{c}\text { 14:00 } \\
\text { Baixa-mar }\end{array}$ & $\begin{array}{c}17: 00 \\
\text { Enchente }\end{array}$ \\
\hline Favella ehrenbergï & 562 & 187 & 187 & 187 & - & - & - & - & - & - \\
\hline Brachionus plicatilis & 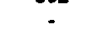 & 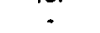 & 1875 & $\mathbf{5 2 5 0}$ & 187 & 987 & 375 & 1312 & 375 & 375 \\
\hline Larvas de Polychaeta & 187 & 375 & 187 & 3562 & 937 & 187 & 187 & 187 & - & 375 \\
\hline Larvas de Bivalvia & 187 & - & - & 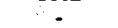 & - & - & - & - & - & - \\
\hline Larvas de Gastropoda & 187 & . & 375 & 187 & 1500 & - & - & 187 & - & - \\
\hline Náuplios de Cirripedia & 2812 & 6000 & 375 & 375 & 19875 & 375 & - & 375 & 375 & - \\
\hline Cipris de Cirripedia & 187 & 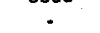 & 375 & 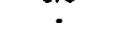 & 187 & - & . & - & - & 187 \\
\hline Penilia avirostris & - & - & $\because$ & . & - & - & 187 & - & - & - \\
\hline Moina sp & - & - & 187 & - & - & 562 & 375 & 187 & - & 187 \\
\hline Náuplios de Copepoda & 937 & 375 & 187 & 1312 & 5812 & 375 & 375 & 375 & 562 & 187 \\
\hline Eucalanus pileatus $\left(0^{*}\right)$ & 187 & 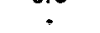 & $\because$ & - & - & - & - & $\because$ & - & - \\
\hline Eucalanus pileatus $(Q)$ & 187 & - & $\cdots$ & - & 187 & $=$ & - & - & - & - \\
\hline Paracalanus quasimodo $\left(\sigma^{*}\right)$ & 187 & - & - & - & 187 & - & • & - & - & - \\
\hline Paracalanus quasimodo ( $($ ) & 937 & - & - & - & 937 & - & - & 187 & - & - \\
\hline Paracalanus indicus $\left(\sigma^{+}\right)$ & - & . & - & - & 187 & - & - & - & - & - \\
\hline Paracalanus indicus (O) & 750 & . & - & - & 187 & - & . & 187 & - & - \\
\hline Paracalanus crassirostris $\left(0^{\prime}\right)$ & 187 & - & - & . & 187 & - & - & 187 & - & - \\
\hline Paracalanus crassirostris (Q) & 187 & 187 & 187 & - & 187 & - & - & 375 & - & - \\
\hline Centropages velificatus $\left(0^{\prime}\right)$ & 187 & $\uparrow 87$ & $=$ & - & - & - & - & - & - & - \\
\hline Centropages velificatus $(8)$ & - & 375 & - & - & 187 & - & - & - & - & - \\
\hline Notodiaptomus cearensis $\left(\delta^{\prime}\right)$ & . & - & - & - & - & - & - & 187 & - & - \\
\hline Pseudodiaptomus acutus (o') & - & 187 & - & . & 187 & - & . & 167 & - & 187 \\
\hline Pseudodiaptomus acutus (Q) & - & 187 & 187 & - & - & - & - & . & - & - \\
\hline Labidocera fuviatilis $\left(0^{\circ}\right)$ & . & - & - & . & 187 & - & - & . & - & - \\
\hline Labidocera fuviablis (Q) & - & 187 & - & - & - & - & - & - & - & - \\
\hline Acartia illjeborg $\left(0^{*}\right)$ & 562 & . & - & - & 937 & - & - & - & - & - \\
\hline Acartia hlljeborg (Q) & 937 & - & - & - & 1500 & - & - & 187 & - & - \\
\hline Oithona osvaldocnuzi $\left(\sigma^{*}\right)$ & 1875 & 375 & 187 & 187 & 2062 & 187 & . & 562 & 375 & 187 \\
\hline Oithona osvaldocruzi ( 8 ) & 10312 & 1312 & 187 & 187 & 13875 & 375 & 750 & 1125 & 562 & 187 \\
\hline Oithona hebes $\left(0^{\circ}\right)$ & 187 & 562 & 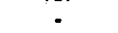 & 187 & - & - & 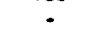 & 187 & - & - \\
\hline Oithana hebes ( 8 ) & 375 & 750 & 187 & 967 & 2250 & 375 & 562 & 187 & . & - \\
\hline Hemicyclops thalassius ( $\left.0^{*}\right)$ & - & - & - & 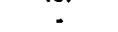 & 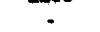 & - & 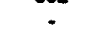 & 187 & - & 187 \\
\hline Hemicyclops thalassius $(Q)$ & 375 & - & 562 & - & 187 & 187 & - & 562 & 187 & 187 \\
\hline Euterpina acuttrons $\left(\sigma^{\prime}\right)$ & 187 & - & 187 & - & . & - & - & - & - & - \\
\hline Euterpina acutfrons (Q) & 375 & 187 & 375 & 187 & 375 & 187 & 375 & 187 & 187 & 375 \\
\hline Sagitta sp & 375 & 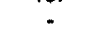 & - & 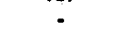 & 187 & 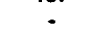 & . & - & 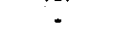 & - \\
\hline Larvas de Crustacea Decapoda & - & - & . & - & . & - & - & 187 & - & + \\
\hline Zoeas de Brachyura & 1500 & 375 & 375 & 187 & 187 & - & - & 552 & - & 187 \\
\hline Ovos de Peixes & 187 & - & 187 & - & - & - & . & - & - & - \\
\hline Larvas de Peixes & - & - & 187 & . & - & - & - & 187 & - & - \\
\hline TOTAL HOLOPLÁNCTON & 19868 & 5058 & 4995 & 7684 & 29992 & 2435 & 2999 & 6368 & 2248 & 2059 \\
\hline TOTAL MEROPLANCTON & 5247 & 6750 & 2061 & 4311 & 22686 & 562 & 187 & 1685 & 375 & 749 \\
\hline TOTAL & 25115 & 11808 & 6556 & 11995 & 52678 & 2997 & 3186 & 8053 & 2623 & 2808 \\
\hline DIVERSIDADE (BITS/IND) & 2.91 & 2.47 & 3.62 & 2.23 & 2.60 & 3.03 & 2.86 & 3.73 & 2.35 & 3.24 \\
\hline EQUITABILIDADE & 0.67 & 0.68 & 0.88 & 0.67 & 0.59 & 0.95 & 0.95 & 0.87 & 0.91 & 0.97 \\
\hline
\end{tabular}




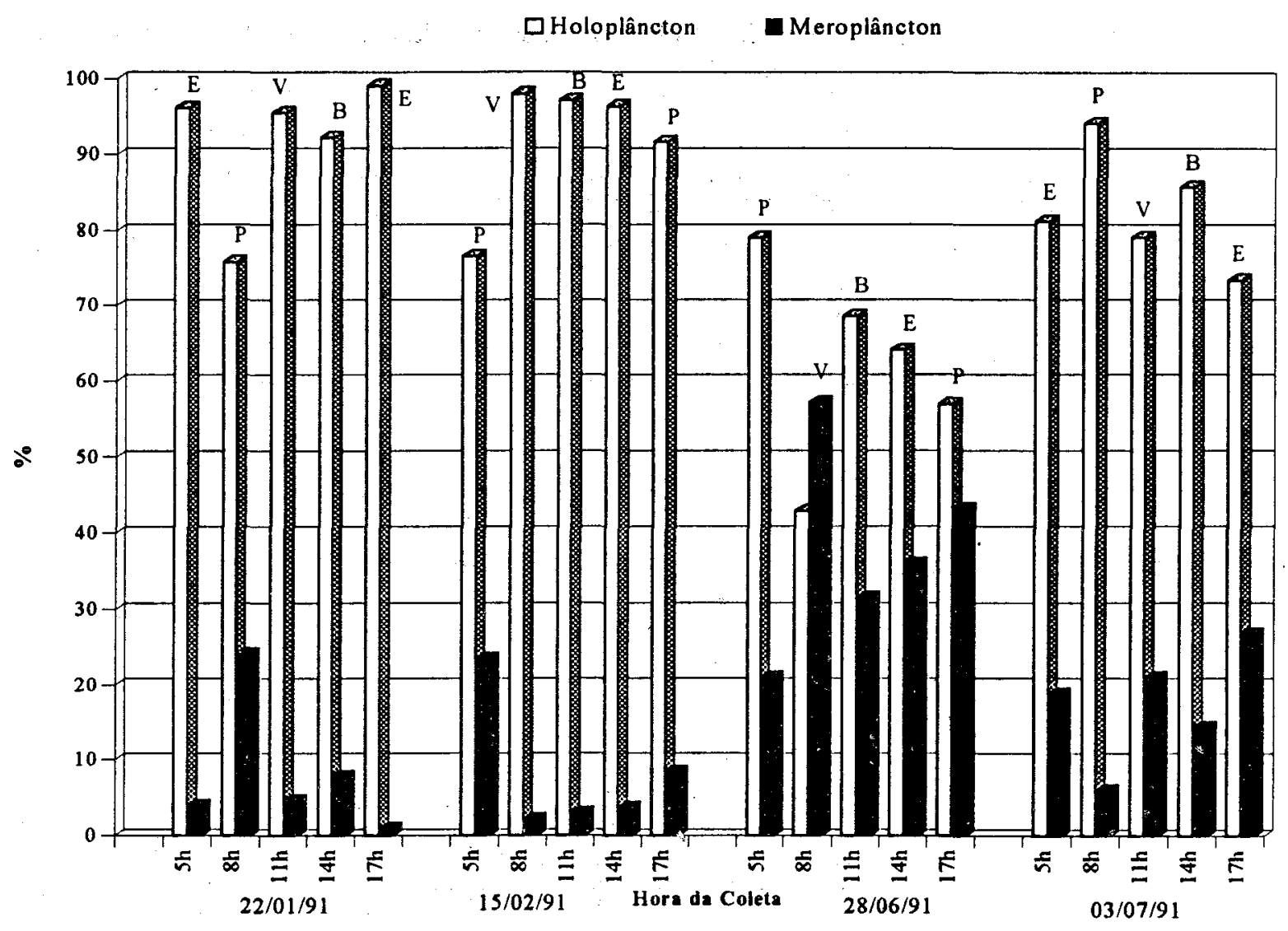

Fig. 2. Variação percentual do holo e meroplâncton no estuário do Pina (PE). $P=$ preamar; $E=$ enchente; $V=$ vazante; $B=$ baixa-mar.

No período chuvoso, foi registrado um declínio das taxas quantitativas do zooplâncton, com o valor máximo de 52.678 org. $\mathrm{m}^{-3}$ em junho, durante a maré cheia. Os organismos holoplanctônicos apresentaram densidades reduzidas, com um máximo de 29.992 org. $\mathrm{m}^{-3}$ em junho (maré cheia). Entre estes organismos destacou-se Oithona osvaldocruzi (Q) (máximo de 13.875 org. $\mathrm{m}^{-3}$ ).

Nesse período sazonal, o meroplâncton mostrou taxas quantitativas mais significativas. Náuplios de Cirripedia foram os organismos meroplanctônicos com contribuição percentual mais significativa, constituindo até $50,80 \%$ do zooplâncton total durante a maré vazante (junho às 08:00 h).

A diversidade específica apresentou valores médios $( \pm$ 2,0bits.ind. ${ }^{-1}$ ) durante o período seco, e foi um pouco mais elevada durante o período chuvoso (máximo de 3,73 bits.ind $^{-1}$ ). A diversidade mínima encontrada foi de 1,36 bits.ind. ${ }^{-1}$, às 05:00 $\mathrm{h}$ em janeiro (maré enchente). Durante o período seco, a diversidade de espécies aumentou durante as marés enchente e preamar, reduzindo-se durante as marés vazante e baixa-mar. No período chuvoso, esse padrão de varição horária não foi observado.

A eqüitabilidade das amostras variou de um máximo de 0,97 (17:00 h em julho, durante a maré enchente) a um mínimo de 0,39 $(05: 00 \mathrm{~h}$ em janeiro, também na maré enchente).

Os taxa utilizados na análise de "cluster" distribuíram-se em 5 (cinco) agrupamentos principais (Fig. 4), os quais estiveram constituídos por organismos predominantemente costeiros, eurialinos e euritérmicos, bem adaptados a áreas estuarinas, sendo eles:

\section{Grupo 1:}

Centropyxis acureata, Larvas de Crustacea Decapoda e Acartia lilljeborgi

Este grupo apresentou a similaridade máxima de 0,67 entre Centropyxis acureata e Larvas de Crustacea Decapoda. Estes organismos mostraram ocorrência limitada na área, com preferência por águas mais turvas. 


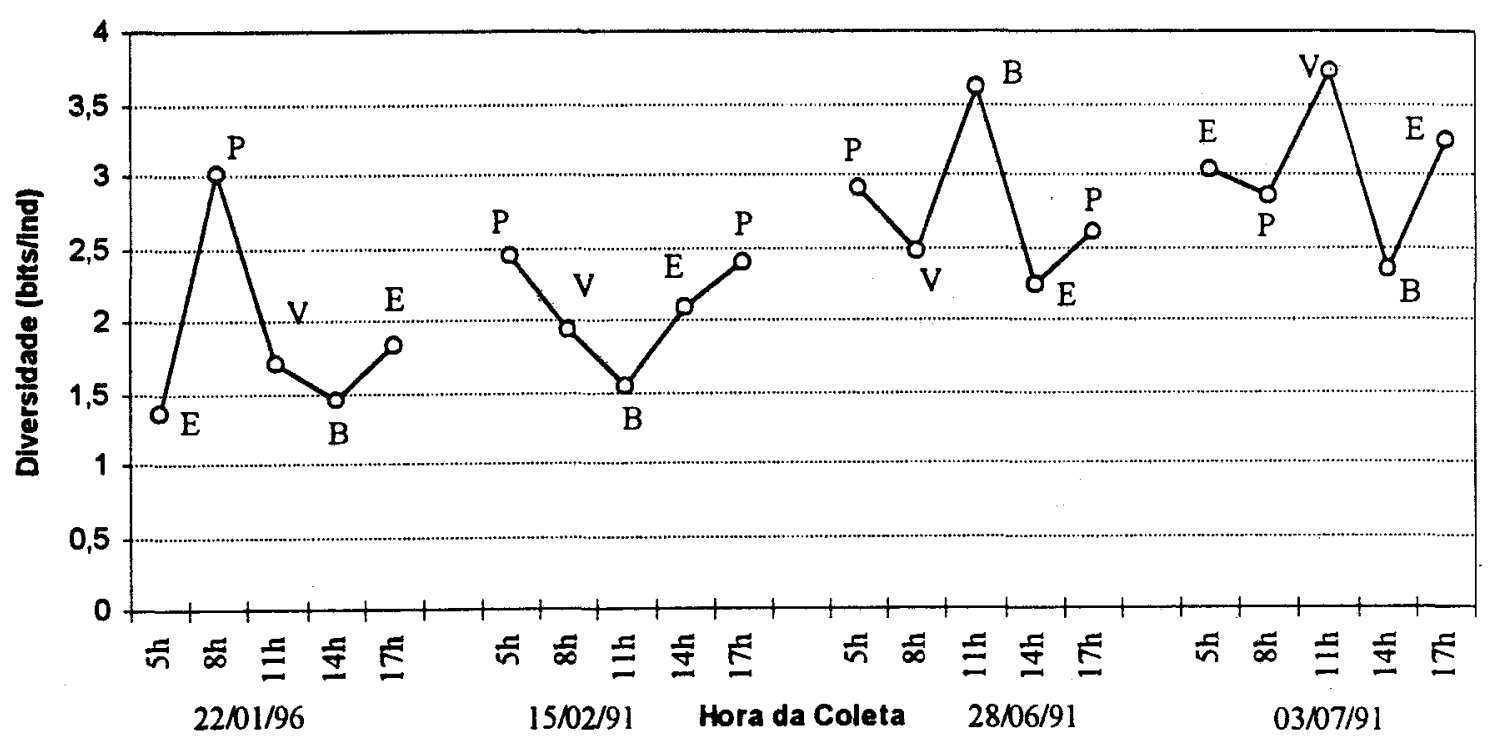

Fig. 3. Distribuição temporal da diversidade de espécies (bits/ind) no estuário do Pina.

Índice de Sorensen

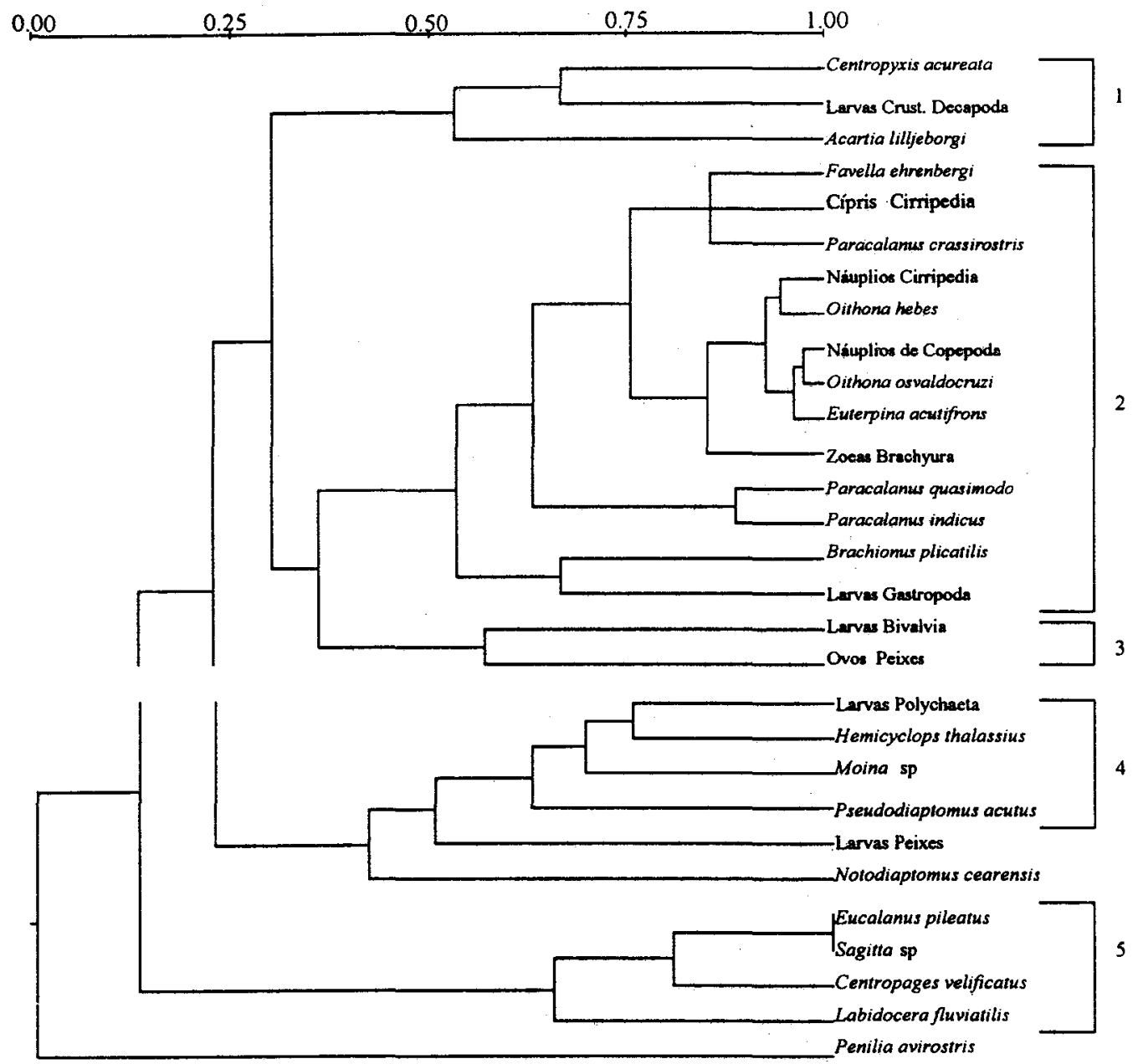

Fig. 4. Dendograma de similaridade do zooplâncton do estuário do Pina (PE). 


\section{Grupo 2:}

Favella ehrenbergi, Cípris de Cirripedia, Paracalanus crassirostris, Náuplios de Cirripedia, Oithona hebes, Náuplios de Copepoda, Oithona osvaldocnizi, Euterpina acutifrons, Zoeas de Brachyura, Paracalanus quasimodo, Paracalanus indicus, Brachionus plicatilis, Larvas de Gastropoda.

O grupo reuniu os organismos residentes no estuário, cuja maioria é de origem marinha, caracterizado principalmente pelas interações tróficas entre seus constituintes.

\section{Grupo 3:}

Larvas de Bivalvia e Ovos de Peixes

$O$ índice de similaridade entre esses organismos foi baixo $(0,57)$. Esse agrupamento reuniu larvas meroplanctônicas freqüentemente encontradas em ambientes estuarinos.

\section{Grupo 4}

Larvas de Polychaeta, Hemicyclops thalassius, Moina sp e Pseudodiaptomus acutus

Esse grupo englobou organismos com preferência por águas de menor salinidade e mais turvas, que ocorreram no estuário do Pina apenas durante o período chuvoso.

\section{Grupo 5:}

Eucalanus pileatus, Sagitta $\mathrm{sp}$, Centropages velificatus $\mathrm{e}$ Labidocera fluviatilis

Os organismos constituintes desse grupo caracterizam-se por habitar preferencialmente águas costeiras, sendo trazidos para o estuário pelas correntes de marés.

\section{Discussão}

Os ambientes estuarinos são caracterizados pela variabilidade de seus componentes ambientais, o que os torna ambientes altamente seletivos para fauna e flora. Em funçāo disto, o zooplâncton de baías e estuários caracteriza-se pela dominância numérica de uma ou poucas espécies que apresentam altas taxas de abundância (Perkins, 1974; Kimmerer \& Mckinnon, 1987 a e b).

No estuário do Pina, foi verificado o domínio quantitativo dos Copepoda, principalmente Oithona osvaldocruzi, Oithona hebes, Paracalanus crassirostris e Paracalanus quasimodo. Essas espécies, segundo Björnberg (1981) são euritérmicas e eurialinas, tendo sua distribuição mais freqüente em águas costeiras e estuarinas.

Foram comuns também "picos" temporais de Favella ehrenbergii e Brachionus plicatilis, especialmente durante o período seco (janeiro e fevereiro). Pulsos quantitativos do microzooplâncton em águas costeiras também já foram registrados por Hernroth (1983) e Turner et al. (1989).

O microzooplâncton é considerado como o maior consumidor de nanofitoplâncton. Assim, aumentos da população microzooplanctônica acompanham o "bloom" fitoplanctônico, sugerindo que a abundância do zooplâncton estuarino pode ser controlada em parte pela disponibilidade de alimento (Pierce \& Turner, 1992; Buskey, 1993).

Entretanto, Turner \& Tester (1992), mostraram que existem fortes indícios de que rotíferos (incluindo Brachionus plicatilis) e tintinídeos do gênero Favella sejam potencialmente bacterívoros, i.e., alimentam-se também de bactérias presentes na coluna d'água, constituindo assim, um importante elo da cadeia microbiana em sistemas aquáticos, transferindo a produção bacteriana a níveis tróficos mais altos na cadeia alimentar. Feitosa (1988), relata para o estuário do Pina valores de colimetria (N.M.P.) entre 200 a $30.000 \mathrm{org} / 100 \mathrm{ml}$, oriundos provavelmente da descarga de resíduos orgânicos na área, o que pode constituir uma fonte alimentar abundante para o microzooplâncton.

O meroplâncton esteve representado por vários taxa, distribuídos de maneira irregular nos períodos sazonais estudados. As condições ambientais características de cada período sazonal, determinam os processos de reprodução das comunidades bentônicas e nectônicas, influindo na distribuição das larvas meroplanctônicas (Williams \& Collins, 1986; Tundisi, 1970; Soetaert \& Van Rijswijk, 1993). Alguns organismos, como as larvas de Polychaeta, apresentaram uma sazonalidade acentuada, ocorrendo apenas durante o período chuvoso.

A variação diurna no estuário do Pina mostrou que os padrões de dominância de uma ou outra espécie, podem ser rapidamente alterados em função das diferentes correntes de marés. Outros processos como alimentação e dispersão, também parecem influir nessas variações. 
As condições hidrográficas em um estuário - descarga de rios, marés, processos de mistura, etc - afetam bastante a distribuição do zooplâncton (Kennish, 1990). As correntes podem deslocar populações zooplanctônicas, alterando assim a densidade no corpo principal do estuário.

A influência das condições hidrográficas de sistemas estuarinos sobre o comportamento de migração vertical em organismos zooplanctônicos, vem sendo estudada (Norcross \& Shaw, 1984; Paffenhöfer et al., 1989; Lam Hoai, 1991; Checkley et al., 1992; Xiao \& Greenwood, 1992). Os organismos zooplanctônicos parecem responder a esses fatores, adotando estratégias de manutenção em áreas adequadas ao seu desenvolvimento ou deslocando-se horizontalmente no estuário.

Neumann-Leitão et al. (1992 a e b) realizaram trabalhos de variação diurna em estuários de Pernambuco, os quais mostraram ocorrer variações quantitativas muito significativas do zooplâncton, em relação aos diferentes horários de coleta, em decorrência da dinâmica das marés. Variaçōes diurnas do zooplâncton já foram registradas para outras áreas estuarinas (Nascimento-Vieira e Eskinazi-Sant'Anna, 1987/89; Peterson, 1986).

Dentre os organismos residentes no estuário do Pina destacaram-se Oithona osvaldocruzi e Oithona hebes. Estudos realizados por Ohman (1990), mostram que algumas espécies do gênero Oithona não apresentam comportamento migratório, adotando estratégias de manutenção na camada superficial da coluna d'agua.

Além da variação diurna do zooplâncton foram registradas flutuaçōes quantitativas sazonais bem marcadas. Durante o período seco, o zooplâncton apresentou taxas quantitativas mais elevadas, especialmente durante o mês de fevereiro. Os meses de junho e julho caracterizaram-se pela redução numérica do zooplâncton, particularmente durante o mês de julho, onde foram registrados as densidades mais reduzidas.

A diversidade específica no estuário do Pina apresentou valores médios, sendo os valores mais elevados encontrados no período chuvoso. Este fato evidencia processos de seleção de espécies na área, restringindo através de limites abióticos (temperatura, salinidade, oxigênio dissolvido, etc) e bióticos (disponibilidade de alimentos, predação), a ocorrência dos organismos zooplanctônicos mais sensíveis às variações ambientais.

Quanto à variação diurna, a diversidade foi mais alta durante as preamares e reduzida durante as marés vazante e baixa. No período seco, foi encontrada uma correlação positiva da diversidade específica com a salinidade $\left(\mathrm{r}^{2}=\right.$ $0,67)$, mostrando a influência das marés na distribuição das espécies no estuário.
No período chuvoso, essa correlação foi negativa $\left(\mathrm{r}^{2}=\right.$ $-0,06)$, estando a diversidade associada ao transporte de organismos pelo fluxo de água doce. Contribuíram para o aumento da diversidade no período chuvoso; ocorrências ocasionais de copépodos, quando espécies de preferências limnéticas como Notodiaptomus cearensis e Pseudodiaptomus acutus e de hábitos mais bentônicos, como Hemicyclops thalassius foram encontradas, além das larvas de poliquetas.

Este mesmo padrão estrutural foi encontrado por Neumann-Leitão et al. (1992 a e b), em estuários de Pernambuco, quando a diversidade foi mais elevada durante o período chuvoso.

$\mathrm{O}$ zooplâncton agrupou-se principalmente de acordo com suas relações tróficas (herbívoros, omnívoros ou carnívoros), estágios de desenvolvimento (larvas) e seletividade ambiental (eurialinas, euritérmicas etc).

Segundo Petipa (1979) e Lopes (1994), as correlações entre grupos zooplanctônicos baseiam-se principalmente nas relaçōes tróficas. Assim, larvas meroplanctônicas são freqüentemente associadas ao microzooplâncton (especialmente tintinídeos), uma vez que estes também constituem parte de sua dieta principal (Stoecker \& Egloff, 1987), caracterizando as relaçōes tróficas existentes entre a abundância e ocorrência dos organismos zooplanctônicos.

\section{Agradecimentos}

Os autores agradecem à FAPESP pelo apoio financeiro a este trabalho (Proc. 90/1478-8). À Dra Tagea K. S. Björnberg e Dra Sigrid Neumann Leitão pela revisão do manuscrito, críticas e sugestões e aos revisores anônimos pelas valiosas contribuições.

\section{Referências bibliográfícas}

American Public Health Association. 1985. Standard methods for the examination of water and wastewater. $16^{\text {th }}$ ed. New York, APHA. 1268p.

Björnberg, T. K. S. 1981. Copepoda. In: Boltovskoy, D., ed. Atlas del zooplancton del Atlantico Suddocidental y métodos de trabajo com el zooplancton marino. Mar del Plata, INIDEP. 936 p. 
Buskey, E. J. 1993. Annual pattern of microand mesozooplankton abundance and biomass in a subtropical estuary. J. Plankt. Res., 15 (8): 907-924.

Checkley, Jr, D. M.; Uye, S.; Dagg, M. J.; Mullin, M. M.; Omori, M.; Zhu, M. Y. 1992. Diel variation of the zooplankton and its environment at neritic stations in the Inland Sea of Japan and the northwest Gulf of Mexico. J. Plankt Res., 14 (1):1-40.

CONDEPE. Instituto de Desenvolvimento de Pernambuco. 1980. Perfil fisiografico das bacias hidrográficas de Pernambuco. Relatório Técnico. 1v. 275p.

Feitosa, F. A. do N. 1988. Producão primária do fitoplâncton relacionada com parâmetros bióticos e abióticos na Bacia do Pina (Recife-Pernambuco, Brasil). Dissertação de Mestrado. Universidade Federal de Pernambuco, Departamento de Oceanografia. 270p.

Hernroth, L. 1983. Marine pelagic rotifers and tintinnids-important trophic links in the spring plankton community of the Gullmar Fjord, Sweden. J. Plankt. Res., 5(1):835-846.

Kennish, M. J. (1990). Zooplankton. In: Kennish, M. J., ed. Ecology of Estuaries. Biological Aspects. Florida, CRC Press. v.2, p. 111-153.

Kimmerer, W. J.; Mckinnon, A. D. 1987 a. Zooplankton in a marine bay. I. Horizontal distribution used to estimate net population growth rates. Mar. Ecol.-Progr. Ser., 41:43-52.

Kimmerer, W. J.; Mckinnon, A. D. 1987 b. Zooplankton in a marine bay. II. Vertical migration to maintain horizontal distributions. Mar. Ecol.-Progr. Ser., 41:53-60.

Lam Hoai, T. 1991. Zooplankton counted by image analysis and size-frequency distributions in a coastal lagoon. Arch. Hydrobiol., 121(2):147-159.

Lopes, R. M. 1994. Zooplankton distribution in the Guaraú river estuary (South-eastern Brazil). Estuar. coast. Shelf Sci., 39: 287-302.
Nascimento-Vieira, D. A.; Eskinazi-Sant'Anna, E. M. 1987/89. Composição do Zooplâncton no Estuário do Rio Timbó (Pernambuco-Brasil). Trabhs Oceanogr., Univ. Fed. Pernambuco, 20:77-98.

Neumann-Leitão, S.; Gusmão, L. M. de O.; Nascimento-Vieira, D. A. 1992 a. Zooplâncton dos estuários dos rios Massangana e Tatuoca, Suape (PE-Brasil) . Arq. Biol. Tecnol., 35(2):341-360.

Neumann-Leitão, S.; Paranaguá, M. N.; Valentim, J. 1992 b. The planktonic rotifers of the estuarine lagunar complex of Suape (Pernambuco, Brasil). Hydrobiologia, 232:133-143.

Norcross, B. L.; Shaw, R. F. 1984. Oceanic and estuarine transport of fish eggs and larvae: a review. Am. Fis. Soc. Symp., 8:153-165.

Ohman, M. D. 1990. The demographic benefits of diel migration by zooplankton. Ecol. Monogr., 60(3); $257-281$

Paffenhöfer, G. A.; Huntley, M. E.; Davis, C. S. 1989. Future marine zooplankton research-a perspective. Marine Zooplankton Colloquium. Mar. Ecol.- Prog. Ser., 55:197-206.

Parsons, T. R.; Takahashi, M.; Hargrave, B. 1984. Biological oceanographic processes. $3^{\text {th }}$ ed. Great Britain, Pergamon Press. 330p.

Perkins, E. J. 1974. The biology of estuaries and coastal waters. London, Academic Press. 678p.

Peterson, W. T. 1986. The effects of seasonal variations in stratification on plankton dynamics in Long Island Sound. Lect. Not. coast. estuar. stud.,17:287-319.

Petipa, T. S. 1979. Trophic relationships in communities and the functioning of marine ecosystems. I.Studies in trophic relationships in pelagic communities of the Southern seas of the USSR and in the tropical Pacific. In: Marine Production Mechanisms. Cambridge, Cambridge University Press. p. 233-250.

Pierce, R. W.; Turner, J. T. 1992. Ecology of planktonic ciliates in marine food webs. Revs aquat. Sci., 6(2):139-181. 
Poole, H. H.; Atkins, W. R. G. 1929. Photo-electric measurements of submarine illumination throught the year. J. mar. biol. Ass. U. K., 16:297-324.

Shannon, L. E. 1948. A mathematical theory of communication. Bull. Sys. Technol. J.,27:379-423.

Soetaert, K.; Van Rijswijk, P. 1993. Spatial and temporal patterns of the zooplankton in the Westerschelde estuary. Mar. Ecol.-Prog. Ser., 97: 47-59.

Sorensen, T. 1948. A method for establishing groups of equal amplitude in plant sociology based on similarity of species content and its application to analysis of the vegetation on Danish commons. Biol. Skr., 5(4):1-34.

Stoecker, D.; Egloff, D. A. 1987. Predation by Acartia tonsa Dana on planktonic ciliates and rotifers. J. expl mar. Biol. Ecol., 110:53-68.

Strickland, J. D. H.; Parsons, T. R. 1965. A manual of seawater analysis. Bull. Fish. Res. Bd Can.,125:1-205.

Tundisi, J. G. 1970. O plâncton estuarino. Contrções Inst. oceanogr. Univ. S. Paulo, Série Oceanografia Biológica, 19:1-22.

Turner, J. T.; Borkman, D. G.; Lima, W.; Pierce, R. W. 1989. A seasonal study of plankton, larval fish, and water quality in Buzzards Bay, Massachussetts. Interim Data Report, October, 1987 through September, 1988. Massachussets Department of Environmental Quality Engineering (DEQE), p. 1-48.
Turner, J. T.; Tester, P. A. 1992. Zooplankton fceding ecology: bacterivory by metazoan microzooplankton. J. expl mar. Biol. Ecol., 160: 149-167.

UNESCO. 1973. International Oceanographic Table. Britain Wormly, 2:141p.

Williams, R.; Collins, N. R. 1986. Seasonal composition of meroplankton and holoplankton in the Bristol Channel. Mar. Biol., 92:93-101.

Xiao, Y.; Greenwood, G. G. 1992. Distribution and behaviour of Acetes sibogae Hansen (Decapoda, Crustacea) in an estuary in relation to tidal and diel environmental changes. J. Plankt. Res., 14(3):393-407.

(Manuscrito recebido 21 novembro 1994; revisto em 02 outubro 1995; aceito 20 maio 1996) 